\title{
Assessment of Rational Use of Antibiotics in a Private Tertiary Care Teaching Hospital
}

\author{
B. Rajalingam, Achsah Susan Alex, Adreen Godwin*, Chinnu Cherian, Clincy Cyriac
}

Department of Pharmacy Practice, College of Pharmacy-Sri Ramakrishna Institute of Paramedical Sciences, Sri Ramakrishna Hospital Campus, 395, Sarojini Naidu Road, Coimbatore-641044, INDIA.

\begin{abstract}
Introduction: Rational use of antibiotics is important, as antibiotic resistance is not only a problem for the individual patient but also reduces effectiveness of established treatment and has become a major threat to public health by increasing complexity, cost of treatment and reducing the probability of successful outcome. Objectives: To compare the treatment of different diseases with standard clinical guidelines by implementing indicators for antibiotic usage. Methods: 200 patients were enrolled in a 6 month prospective randomised study as per the inclusion and exclusion criteria. Results: A total of 1955 general drugs were prescribed to patients with an average of $9.09 \pm 6.95$ drugs. The average length of stay for study population was found to be $7.5 \pm 4.18$ days. The rationality was assessed using guidelines prepared from Micromedex and NFI 2011. The most commonly prescribed antibiotics were Ceftriaxone (15.38\%) and Levofloxacin $(6.76 \%)$. The most commonly prescribed FDC were Cefepime + Tazobactum (19.69\%) and Piperacillin + Tazobactum (14.76\%). Sensitivity tests were done for 37 patients and was found that Cefepime/Tazobactum were more sensitive to S. pneumoniae followed by gram positive cocci and Amikacin to be more sensitive to E.coli. When rationality was checked for the overall appropriateness of antibiotics, it was found to be $57.85 \%$. Appropriateness of antibiotics was checked for indication (60.61\%), duration (55.38\%), dose (59.07\%) and frequency (56.31\%). The average interaction per prescription was found to be $1.5 \pm 1.29$. Out of 205 incompatibilities $60.97 \%$ were with antibiotics. Conclusion: There is a vital need for microbiological investigation before treatment of infections. This study should help physicians to have better insight about prescription patterns.
\end{abstract}

Key words: Rationality, Antibiotics, Micromedex, Drug Interactions, IV incompatibility.

\section{INTRODUCTION}

The rationality of antibiotics are the most controversial and debated issue in today's clinical practice. ${ }^{1}$ Irrational antibiotics/antibacterials $(\mathrm{ABs})$ usage is a global problem especially in developing countries resulting in an increased emergence of resistance to most common bacteria, higher cost of treatment, prolonged hospitalization and adverse drug reactions. ${ }^{2}$ Promoting the rational use of medicines would definitely help mankind to fight the disease and the illnesses for a better tomorrow. ${ }^{3}$ Antimicrobials can obviously be important or even lifesaving in appropriate situations, but it is just as important to prevent unnecessary use of antimicrobials which can lead to resistance. ${ }^{4}$ Rational use of drugs requires that patient receive medications appropriate to
Submitted date : 09/12/2015 Accepted date : 13/01/2016

their clinical needs, in doses that meet their own individual requirements for an adequate period of time, at the lowest cost to them and their community. ${ }^{5}$ Irrational use of antibiotics should be checked as injudicious use which can adversely affect the patient; cause emergence of antibiotic resistance and will increase the cost. ${ }^{6}$ The reasons for irrational use are varied comprising of non availability of medicines, self medication, irrational prescribing by the health care professionals and lack of drug information. ${ }^{7}$ World Health Organization WHO estimates that more than half of all medicines are prescribed, dispensed or sold inappropriately and that half of all patients fail to take them correctly. ${ }^{8}$ In 1981, the established the Action Programme on Essential Drugs to
DOI: 10.5530/ijopp.9.1.4

Address for correspondence:

B. Rajalingam,

Department of Pharmacy

Practice, College of PharmacySri Ramakrishna Institute of Paramedical Sciences,

Sri Ramakrishna Hospital Campus, 395,

Sarojini Naidu Road, Coimbatore-641044, INDIA.

Phone no: +91 9443260932

E-mail: brajalingam@gmail.com

(1)

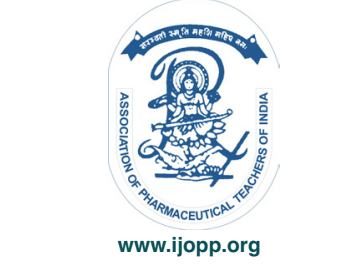


support countries to implement national drug policies and to work towards rational use of drugs. ${ }^{9}$ In India, the Delhi Society for Promotion of Rational Use of Drugs (DSPRUD) was formed to promote the rational use of drugs. ${ }^{10}$ Drug use evaluation is an ongoing systematic process designed to maintain the appropriate and effective use of drugs and also to assess whether drug therapy is rational or not. ${ }^{11}$ WHO and The International Network for Rational Use of Drugs (INRUD) jointly published a standard methodology and prescription indicators for evaluation of drug utilization on different health facilities. ${ }^{12} \mathrm{WHO} /$ INRUD developed drug use indicators(core drug use indicators) to measure the rational use of drugs in primary care. They are prescribing indicators, patient care indicators and facility indicators. ${ }^{13}$ Antibiotics Smart Use [ASU] introduced in 2007 was an innovative model to us for promoting rational use of medicines and counteracts antimicrobial resistance. ${ }^{14}$ Our main objectives were to analyse the rational use of antibiotics using Micromedex and NFI and also to report the irrational use of antibiotics.

\section{MATERIALS AND METHODS}

The study was conducted for a period of 6 months in General Medicine and Pulmonology departments of 700 bedded multi-speciality hospital. All the inpatients of General Medicine and Pulmonology Departments who were prescribed with atleast one antimicrobial agent were included in the study as per the following inclusion criteria i.e. patients above 12 years with atleast one antimicrobial agent prescribed in their prescriptions and were willing to participate. In-patients below 12 years, pregnant and lactating women, patients getting admitted to ICU and who were not willing to participate in the study were excluded from the study. A total of 200 patients who met the inclusion criteria were selected for the study. Patients were well informed about the study and written informed consent was obtained before including them in the study. Data were collected and recorded in a specially designed data entry format during daily regular ward rounds with senior and junior physicians. A guideline regarding rational use of antibiotics was developed after reviewing relevant literatures, electronic database (Micromedex) and using the National Formulary of India 2011 (NFI) with respect to dose, indication, dose, frequency and duration of antibiotics.

\section{RESULTS}

\section{Demograhic Profile}

Among 200 patients participated in the study, the age ranged between 15-87 years with an average of 49.25 \pm
20.69 years. Maximum were from adulthood (36-65) years. $63 \%(n=126)$ were male and $37 \%(n=74)$ were female. The details are given in Table 1.

\section{Department-wise categorisation and length of stay}

Department of General Medicine accounted for the highest number of prescriptions $\{130(65 \%)\}$ followed by Department of Pulmonology $\{70(35 \%)\}$. Most of the study population were hospitalised for a period of (6-10) days with an average length of (7.5 \pm 4.18$)$ days and the same was listed in Table $2 \mathrm{a}$ and $2 \mathrm{~b}$.

\section{Sensitivity Pattern}

In the study population, only 37 patients did their sensitivity tests. The major organisms identified were Streptococcus pneumoniae, E.coli, Staphylococcus aureus, Klebisella pneumoniae, Streptococcus pyogenes, gram positive cocci in clusters, gram negative rods, E.faecalis. Cefepime/Tazobactum was found to be more sensitive to S.pneumoniae and gram positive cocci. Amikacin was were found to be more sensitive to E. coli. Amoxicillin+Clavulanate were found to be resistant to E. coli and S.aureus and Ciprofloxacin and Cefepime+Tazobactum were found to be resistant to K. pneumoniae.

\section{Antibiotics Prescribed}

$325(16.62 \% \mathrm{n}=1955)$ antibiotics were prescribed and the most commonly prescribed antibiotics were cephalosporins (ceftriaxone 15.38\%), followed by quinolones (levofloxacin 6.76\%). In fixed dose combinations, the most commonly prescribed antibiotics were cefepime+tazobactum (19.69\%) followed by piperacillin+tazobactum $(14.76 \%)$. The most commonly prescribed parentral antibiotics were cefepime+ tazobactum administered for a period of 6-7days and ceftriaxone administered for a period of 4-5 days and the same was listed in Table 3. Assessment of antibiotics using WHO prescribing indicators were listed in Table 4.

\section{Assessment of rationality of antibiotics}

When rationality was checked for overall appropriateness of antibiotics prescribed it was found to be $57.85 \%$. It was found that, $60.61 \%$ antibiotics were appropriate specially with reference to indication and $55.38 \%$ with duration. Similarly $59.07 \%$ with dose, $56.31 \%$ with frequency were found to be appropriate and the same is given in the Figure $1 \mathrm{a}$ and $1 \mathrm{~b}$.

\section{DRUG INTERACTIONS}

The results revealed that there were about and had 135 $(67.5 \%)$ prescriptions which had atleast 1 interaction 
Table 1: Table showing Demographic Profile of patients

\begin{tabular}{cc} 
Properties & Percentage \\
\hline Age group & \\
Adolescent (13-18) & 4.5 \\
Early adulthood (19-35) & 19 \\
Adulthood (36-50) & 26 \\
Late adulthood (51-65) & 31 \\
Young old (66-74) & 11.5 \\
Old (75-84) & 7 \\
Old-old (>85) & 1 \\
(WHO classification) & \\
Gender & \\
Male & 63 \\
Female & 37 \\
\hline
\end{tabular}

Table 2a: Study conducted in different departments of the hospital

\begin{tabular}{cc}
\hline Properties & Percentage \\
\hline General Medicine & 65 \\
Pulmonology & 35 \\
\hline
\end{tabular}

\begin{tabular}{cc}
\hline Table 2b: Length of stay in hospital \\
\hline No of days & Percentage \\
\hline $1-5$ & 41 \\
$6-10$ & 53.5 \\
$11-15$ & 5.5 \\
\hline
\end{tabular}

Table 3: Commonly prescribed antibiotics

\begin{tabular}{cc}
\hline Category of the drug & Total Percentage (\%) \\
\hline Cephalosporins $(n=61)$ & 18.12 \\
Quinolone antibiotics $(n=53)$ & 16.29 \\
Macrolides $(n=31)$ & 9.52 \\
Aminoglycosides $(n=25)$ & 7.41 \\
Carbapenams $(n=4)$ & 2.26 \\
Other antibiotics $(n=3)$ & 0.92 \\
Tetracyclines $(n=2)$ & 0.61 \\
Penicillins $(n=1)$ & 0.3 \\
Fixed dose combinations $(n=145)$ & 44.57 \\
\hline
\end{tabular}

Table 4: Assessment of Drug Use Evaluation Using WHO Prescribing Indicators

\begin{tabular}{cc}
\hline Parameters & Percentage \\
\hline $\begin{array}{c}\text { Average number of drugs per encounter } \\
\text { Percentage of drugs prescribed by generic } \\
\text { name }\end{array}$ & 100 \\
Percentage of drugs with an antibiotic \\
prescribed \\
$\begin{array}{c}\text { Percentage of drugs with an injection } \\
\text { prescribed }\end{array}$ & 100 \\
$\begin{array}{c}\text { Percentage of drugs prescribed from essential } \\
\text { drug list or formulary }\end{array}$ & 99.5 \\
\hline
\end{tabular}

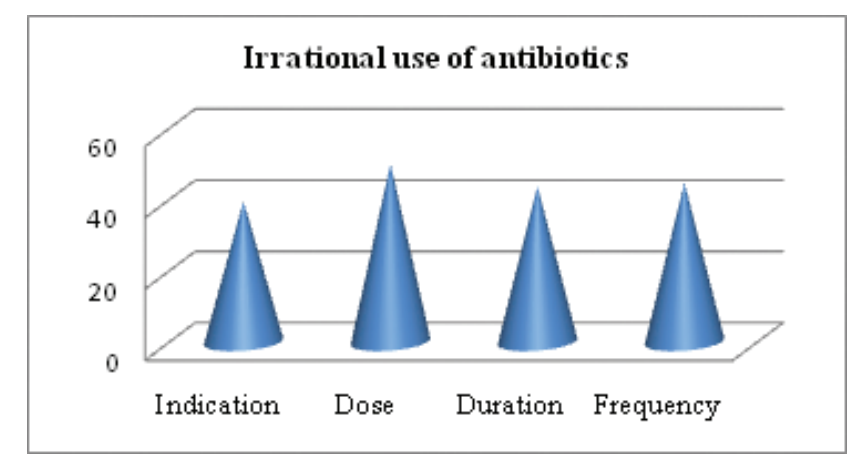

Figure 1b: Irrational use of antibiotics

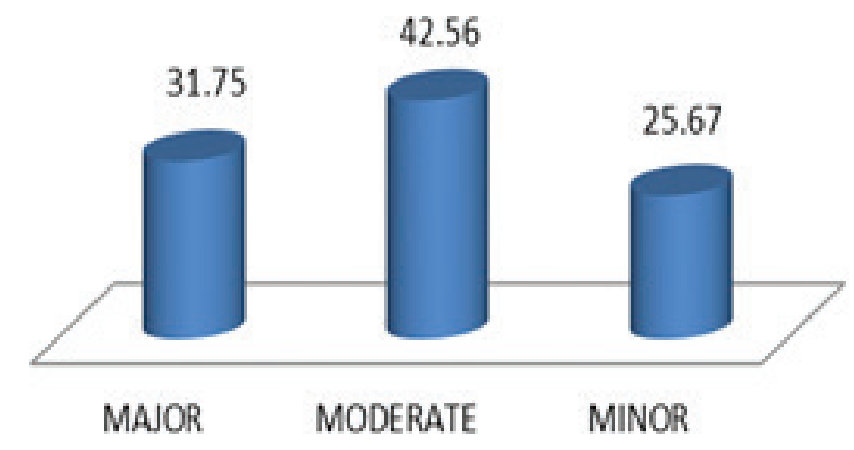

Figure 2: Incidence of Drug interactions $(n=135)$

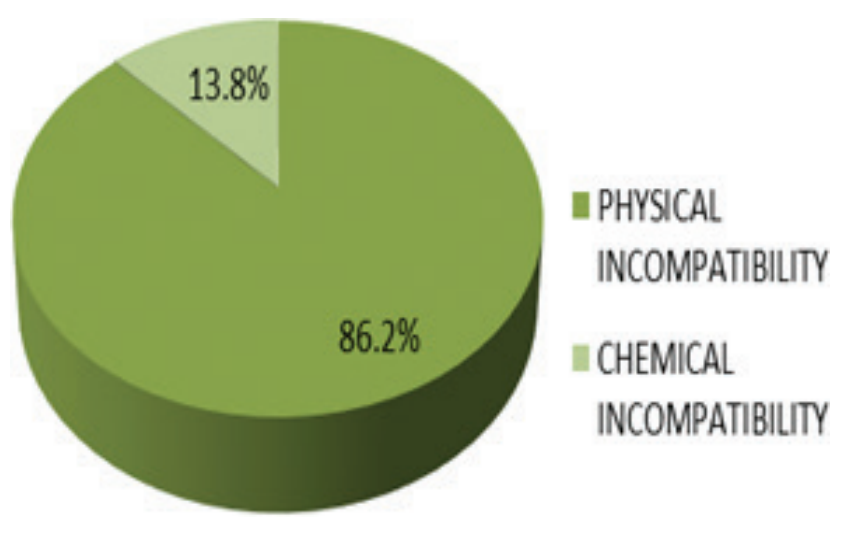

Figure 3: Types of incompatibilities

Indian Journal of Pharmacy Practice, Vol 9, Issue 1, Jan-Mar, 2016 
and remaining 32.5\% $(\mathrm{n}=65)$ did not have any drug interactions at all. Incidence of drug interactions were shown in Figure 2.

Eg. Levofloxacin+Theophylline (Major) : Results in theophylline toxicity. Monitor theophylline levels closely and make appropriate adjustments, when levofloxacin is co-administered.

\section{Compatibility}

The results revealed that $57.5 \%$ of prescription had 1 or more incompatibilities.

\section{Physical incompatibility:}

Cefoperazone+Ondansetron: Cloudiness and particulates developed immediately upon mixing and increased in measured haze or turbidity particulates or colour change was found.

\section{Chemical Incompatibility:}

Cefepime+Theophylline: Chemically unstable. 25\% loss of cefepime occured in $1 \mathrm{hr}$ and the same was shown in Figure 3.

\section{DISCUSSION}

Inourstudy, malepatientswerepredominantand the age of the patients anging ranged between 51-65years were found to be high. The average length of hospital stay was $7.5+$ 4.18 days and can be reduced by appropriate management of the diagnosed diseases so as to reduce the cost needed for the treatment.

The results revealed that out of 200 prescriptions, $325(16.62 \% \mathrm{n}=1955)$ antibiotics were prescribed to the study population. The most commonly prescribed antibiotics were cephalosporins (ceftriaxone 15.38\%), followed by quinolones (levofloxacin 6.76\%). In fixed dose combinations, the most commonly prescribed antibiotics were cefepime+tazobactum (19.69\%) followed by piperacillin +tazobactum $(14.76 \%)$.

$55 \%$ of prescriptions had 1 antibiotic followed by $30 \%$ of prescriptions having 2 antibiotics and the average was found to be $1.625 \pm 0.804$. A similar study was done by Abdel Salam Moahamed Elfaki and reported that $1.6 \pm 0.95$ of antibiotics were prescribed and also found cephalosporins to be used in $1 / 3^{\text {rd }}$ of the prescriptions especially ceftriaxone.

The results revealed that in the study population out of 325 antibiotics prescribed, $268(82.46 \%)$ were administered parentrally and $57(17.54 \%)$ were given as oral. The most commonly prescribed parentral antibiotic were cefepime+tazobactum which was administered for a period of 6-7days and ceftriaxone which was administered for a period of 4-5 days during their complete stay in the hospital. Usually parentral preparation are found more costlier than the oral preparations which was given longer duration and hospitalization is necessary when drug is given as parentral.

The drug use pattern was assessed using WHO prescribing indicators. The range of drugs per encounter was found to be 8-14 and the average was found to be $11.81 \pm$ 5.25. The most commonly prescribed antibiotic by the generic name was found to be ceftriaxone. The most commonly prescribed drugs by the generic name was found to be tramadol, paracetamol and sodium bicarbonate. The results revealed that in the study population 55\% of prescriptions had atleast 1 antibiotic followed by $30 \%$ of prescriptions having 2 antibiotics and the average was found to be $1.625 \pm 0.804$. The results revealed that $25 \%$ of prescriptions had 4 injections followed by $20.5 \%$ prescriptions had 3 injections and the average was found to be $4.16 \pm 1.73$. The results revealed that $43.53 \%$ of drugs were not prescribed from essential drug list and $56.57 \%$ of drugs were prescribed from essential drug list.

The rationality of antibiotics were analysed with the help of guidelines prepared from Micromedex and NFI 2011. The dose, indication, route and duration of antibiotics were verified from Micromedex. The assessment of rationality was performed by collecting evidences from published literature about individual antibiotics. When assessed, 325 antibiotics the results showed that $81.82 \%$ of the individual antibiotics were present (ceftriaxone, levofloxacin, azithromycin, clindamycin) in the Micromedex and $18.18 \%$ was absent in Micromedex (cefotaxime, faropenem). 8 fixed dose combination (piperacillin + tazobactum, cefepime + tazobactum, ofloxacin+ornidazole) of antibiotics prescribed in the study site were absent in the Micromedex. When rationality was checked for overall appropriateness of antibiotics prescribed were analysed and found to be $57.85 \%$. 60.61\% antibiotics were found appropriate specially with reference to indication and $55.38 \%$ with duration. Similarly $59.07 \%$ with dose, $56.31 \%$ with frequency were found to be appropriate.

Prescriptions were subjected for drug interactions. The results revealed that only $67.5 \%(n=135)$ prescriptions had atleast 1 interactions and remaining 32.5\% $(n=65)$ did not have any drug interactions at all. The number of drug interactions from these 135 prescriptions were counted to 148 . The average no. of drug interactions per prescription was found to be $1.5 \pm 1.29$. Out of 200 prescriptions, $25.42 \%$ of interactions were caused by antibiotics. Incidence of drug interactions are major $49(31.75 \%)$, moderate $62(42.56 \%)$ and minor 37 $(25.67 \%)$. 
The IV drugs prescribed in the prescription were analysed for any kind of incompatibility at the time of administration. The results revealed that $57.5 \%$ of prescription had 1 or more incompatibilities. Out of this, $57.5 \%$ the no. of incompatibilities was found to be 205 . When this was further categorised $13.88 \%$ chemical incompatible and $86.2 \%$ were physically incompatible. The results above reveals that out of 205 incompatibilities in 200 prescriptions $60.97 \%$ were found with antibiotics. The average was found to be $1.5 \pm 1.29$.

\section{CONCLUSION}

There is a vital need for microbiological investigation before treatment of infections. This study should help physicians to have better insight about prescription patterns. In comparison with standard guidelines (Micromedex, NFI) deviations of treatment were observed. The results of this study clearly demonstrate that majority of antibiotics were not complying with the criteria developed for the assessment of rationality. It is concluded from the present study that though there is successful combat of infection using antimicrobial agents it is desirable to adopt treatment protocol to increase the success rate.

\section{ACKNOWLEDGEMENTS}

The authors are grateful to all who have contributed to the success of this study. We specially acknowledge the department of General Medicine and Pulmonology of Sri Ramakrishna Hospital, Coimbatore for provision of the data.

It is our privilege and wonderful experience to be part of this esteemed institution and we owe our sincere thanks to our Managing Trustee Thiru. R. Vijayakumar, SNR Sons Trust, Coimbatore.

We are conceited to express our profound gratitude to Dr. P. Sukumaran, M.S., M.Ch., FIACS, Dean, SRH, Coimbatore who had permitted us and provided with the facilities to execute this work.

\section{CONFLICT OF INTEREST}

The author declare no conflict of interest.

\section{ABBREVIATION USED}

NFI: National Fomulary of India
FDC: Fixed Dose Combination
ICU: Intensive Care Unit

\section{REFERENCES}

1. Hanmant A, Priyadarshini K. Prescription analysis to evaluate rational use of antimicrobials. International Journal Of Pharma And Bio Sciences. 2011;2(2)314-9.

2. Bbosa GS, Geoff W, Kyegombe DB, Ogwal-Okeng J. Effects of intervention measures on irrational antibiotics/antibacterial drug use in developing countries: A Systematic Review. Health 6. 2014;6(2):171-87.

3. Igbiks T, Joseph OF. Drug Prescription Pattern in a Nigerian Tertiary Hospital. Tropical Journal of Pharmaceutical Research. 2012;11(1):146-52.

4. Adiveni T, Chandra DS, Sundresh NJ, Kumar BA, Padmini P, Haritha K. Analysing The Rationality Of Antibiotics In Comparing The Different Departments In Rmmch. Journal of Biomedical and Pharmaceutical Research. 2013;2(3):74-6.

5. Farhan AK, Vinod KS, Sanjeevsharma, Preeti SA. Prospective Study on the Antimicrobial Usage in the Medicine Department of a Tertiary Care Teaching Hospital Journal of Clinical and Diagnostic Research. 2013;7(7):1343-6.

6. Upadhyay DK, Palaian S, Shankar PR, Mishra P. Rational Drug Prescribing and Dispensing in Tertiary Care Teaching Hospital of Western Nepal. Journal of Institute of Medicine. 2008;30:233-8.

7. Bhavesh KL, Hiray RS, Ghongane BB. Drug Prescription pattern of Outpatients in a Tertiary Care Teaching Hospital in Maharashtra. Int J Pharm Bio Sci. 2012;3(3):225-9.

8. Vanitha RN, Kannan G, Venkata NM, Vishwakanth D, Nagesh VRD, Yogitha M, Venkata SM, Thennarasu P. A Retrospective Study On Blood Stream Infections And Antibiotic Susceptibility Patterns In A Tertiary Care Teaching Hospital. Int J Pharm Pharm Sci, 2011;4(1):543-8.

9. Essential Drugs. Practical Guidelines, 2013 edition.

10. Sharma NBM, Chaudhary V, Uma A, Jain A, Bansal A. A Prospective Study of Drug Utilization Pattern in Surgery Department in a Tertiary Care Teaching Hospital in Rajasthan. UJP. 2014;3(2):47-50.

11. Hans V. Hogerzeil. Promoting Rational Prescribing: An International Perspective. Br J Clin Pharmac. 2008;39:1-6.

12. Binu M, Rahul S, Surendra K, Hiremath D. Assessment Of Drug Prescribing Practices Using Who Prescribing Indicators In A Private Tertiary Care Teaching Hospital. International Research Journal For Inventions In Pharmaceutical Sciences. 2013;1:26-31.

13. Rajasree G, Pratyusha N, Hiremath D. Assessment Of Drug Dispensing Practices Using Who Patient Care And Health Facility Indicators In A Private Tertiary Care Teaching Hospital. Int J Pharm Pharm Sci. 2013;5(4):368-72.

14. Bulletin of the World Health Organization. 